\title{
Amplification and protein expression of androgen receptor gene in prostate cancer cells: Fluorescence in situ hybridization analysis
}

\author{
XIAN ZHANG, SHI-ZHE HONG, ER-JIANG LIN, DA-YA WANG, ZHI-JIA LI and LI CHEN \\ Department of Urology, Wenzhou Central Hospital, Wenzhou, Zhejiang 325000, P.R. China
}

Received June 16, 2014; Accepted February 24, 2015

DOI: $10.3892 / \mathrm{ol} .2015 .3114$

\begin{abstract}
Prostate cancer (PCa) is the most frequently diagnosed non-skin cancer and the second highest cause of cancer-related mortality in adult males worldwide. PCa is highly dependent upon androgen receptor (AR) signaling for cell proliferation and survival. The AR therefore plays a vital role in the development and function of normal and malignant prostate cells or PCa recurrence. The present study aimed to examine the ubiquity of $\mathrm{AR}$ amplification in $\mathrm{PCa}$ recurrence, even in the absence of androgen. For this purpose, specimens were collected from 37 patients. The amplification of AR and the number of X chromosomes were determined by two-colored fluorescence in situ hybridization analysis. The automated image analysis was used to determine the protein expression of AR. Clinical characteristics and survival in patients whose tumors showed or did not show AR amplification and in X-chromosome polysomy with $\mathrm{PCa}$ recurrence has also been compared. The results showed that $>35 \%$ of patients (13 specimens) exhibited AR amplification. It was also observed that AR was immunostained more intensely in the tumors with amplified AR compared with those tumors with non-amplified AR. This study demonstrated an influential role of AR in tumor growth and progression even after the deprivation of androgen, as well as showing the potential contribution of AR amplification to AR activation even in the relative absence of androgen.
\end{abstract}

\section{Introduction}

The most common malignancy found in American males is prostate cancer ( $\mathrm{PCa}$ ). The disseminated disease is responsible for cancerous mortality and morbidity $(1,2)$. With the introduction and application of the screening for PCa using prostate-specific antigen, the diagnosis of clinically localized

Correspondence to: Mr. Er-Jiang Lin, Department of Urology, Wenzhou Central Hospital, 32 Dajian Lane, Wenzhou, Zhejiang 325000, P.R. China

E-mail: erjianglin507@gmail.com

Key words: amplification, fluorescence in situ hybridization, androgens, survival, immunohistochemistry
PCa has also been increased sharply (3). The use of biologically significant biomarkers may therefore stratify the risk in this group of patients.

Androgen receptor (AR) protein is expressed in nearly all types of PCa, i.e., primary and metastatic (4). The study by Visakorpi et al was the first to report the amplification of the AR gene together with a gain of chromosome $\mathrm{X}$, and suggested a possible mechanism for the progression of PCa following hormone therapy (5). However, the precise mechanism for the association between AR gene amplification and the development of hormone-refractory $\mathrm{PCa}$ remains unknown. Androgens are significant in the growth of normal and malignant prostate cells via AR; the AR binds testosterone or dihydrotestosterone and then stimulates the transcription of androgen-sensitive genes (6). Similar to other proteins in the steroid receptor family, the AR contains separate regions responsible for hormone binding, DNA binding and transcriptional activation (transactivation). AR signaling has been widely studied in the pathogenesis and progression of PCa, the underlying mechanisms of which are believed to involve AR gene amplification, AR gene mutations, elevated AR mRNA or ligand-independent AR activation by growth factors/cytokines. Androgen deprivation therapy is the main therapeutic option for the treatment of advanced cases (7-9). In other words, the AR gene is an important factor in the growth and progression of $\mathrm{CaP}$ and hence, the majority of $\mathrm{PCa}$ is androgen-dependent $(10,11)$. Additionally, in multiple studies, it has been demonstrated that the amplification and overexpression of the AR gene is evident in PCa patients whose tumors are refractory to androgen ablation therapy (12).

The present study compared AR protein expression, and clinical characteristics and survival in patients with PCa whose tumors showed or did not show AR amplification and in X-chromosome polysomy.

\section{Materials and methods}

Specimen source and tissue array. The present study was approved by the Ethics Review Board of Wenzhou Central Hospital (Wenzhou, Zhejiang, China) and all patients provided written informed consent. All samples were collected from Wenzhou Central Hospital between July 2012 and December 2013. A total of 37 male patients were selected by analyzing their medical records. Specimens were collected following detection of an increase in serum prostate-specific 


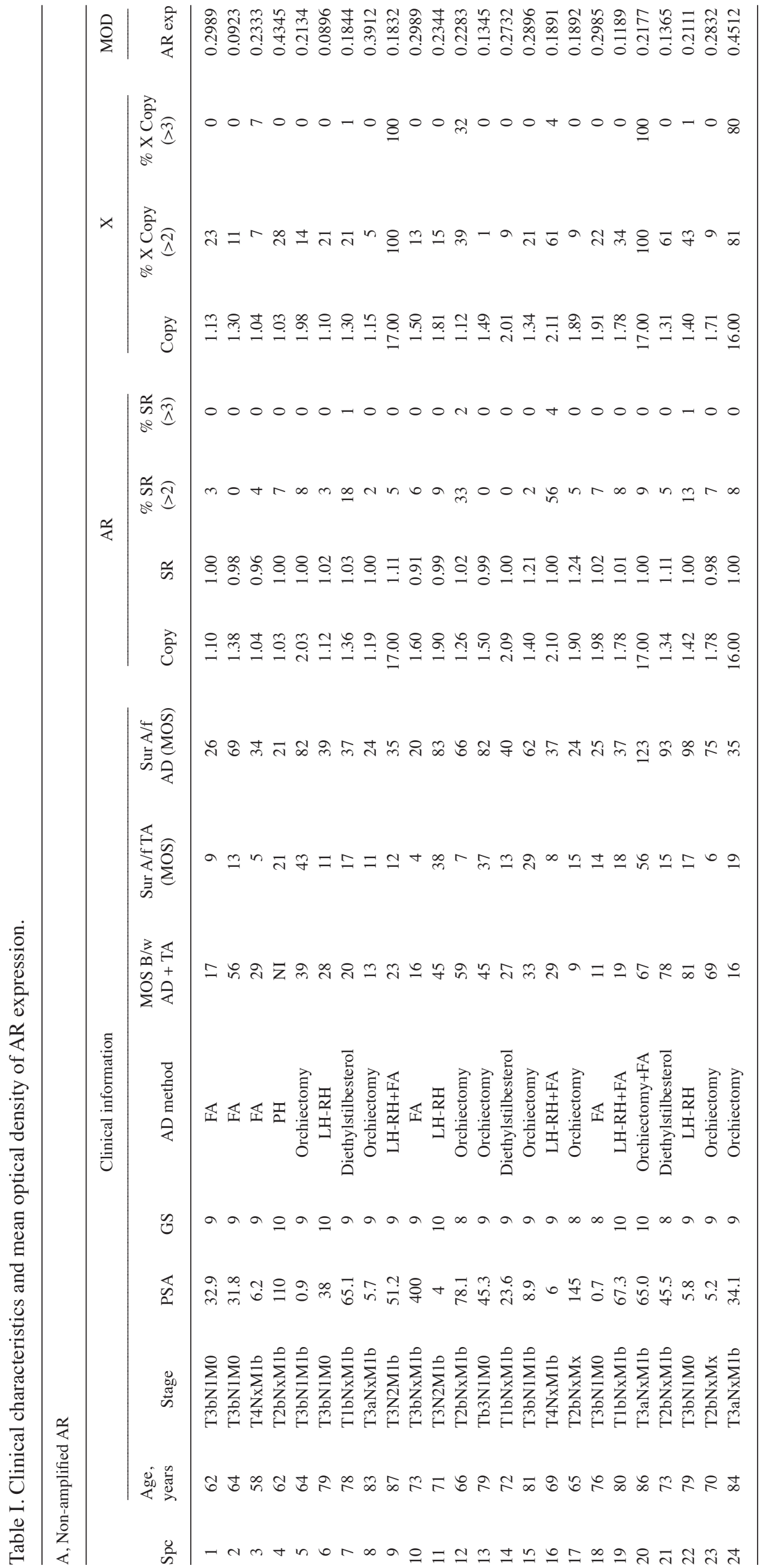

\begin{tabular}{|c|c|c|}
\hline$F$ & $\begin{array}{l}\text { वे. } \\
0 \\
\frac{\alpha}{4}\end{array}$ & 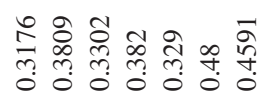 \\
\hline & $\underset{x}{\hat{\tilde{\theta}}} \widehat{x}$ & $00+0000$ \\
\hline$x$ & 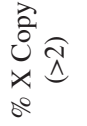 & $n \sigma \infty=\{n \infty$ \\
\hline & 啇 & 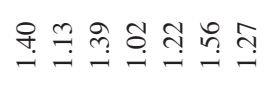 \\
\hline & 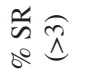 & 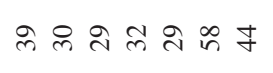 \\
\hline & 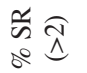 & 宇 f \\
\hline & 芯 & 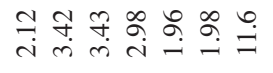 \\
\hline & $\overrightarrow{\hat{\theta}}$ & 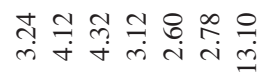 \\
\hline & 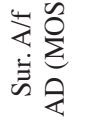 & in in in o \& 83 \\
\hline & $\sum_{i=0}^{0}$ & $a=20=0$ i \\
\hline & $\begin{array}{l}3 \\
0 \\
0+ \\
0 \\
0 \\
0 \\
2\end{array}$ & F F \\
\hline 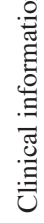 & 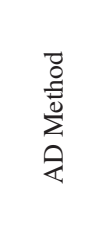 & 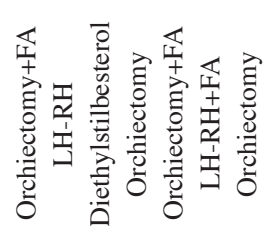 \\
\hline & 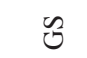 & $\infty \infty a \cap a \subseteq \infty$ \\
\hline & 芯 & 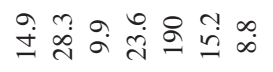 \\
\hline & 80 & 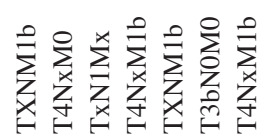 \\
\hline & 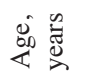 & ㅁㅇ \\
\hline & 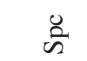 & $\approx 0=\infty 0$ \\
\hline
\end{tabular}


antigen (PSA) level and urinary retention, treated by transurethral resection following androgen deprivation therapy (Table I). The age range of the patients was 58-89 years (Table I).

The tissue array was constructed from 37 duplicate samples of recurrent $\mathrm{PCa}$ and 9 samples of adenofibromyomatous hyperplasia. Tissue preparation artifacts were marked for sampling and 2-mm diameter punch biopsies were obtained. The tissue areas were free of necrosis and cauterization injury. Sections (5- $\mu \mathrm{m}$ thick) were cut from each paraffin block containing a recurrent PCa or adenofibromyomatous hyperplasia sample, and mounted on slides, followed by staining with hematoxylin and eosin, and immunohistochemical analysis.

Immunohistochemical detection. The tissue array sections (BioMax; Guangzhou, China) were used for immunohistochemical detection according to the manufacturer's instructions. Briefly, the sections underwent antigen retrieval in Citra buffer (Biogenex, Shanghai, China) and were cooled to room temperature for pre-incubation with $2 \%$ normal horse serum for $15 \mathrm{~min}$ at $37^{\circ} \mathrm{C}$ followed by incubation with monoclonal anti-human AR antibody (F39.4.1; 1:200; Biogenex) for $1 \mathrm{~h}$. The sections were then treated with biotinylated anti-mouse immunoglobulin $\mathrm{G}(1: 200)$ for $15 \mathrm{~min}$ at $37^{\circ} \mathrm{C}$, followed by avidin-biotin complex amplification (Vector, Beijing, China). The signals were visualized using diaminobenzidine (Vector). The mean optical density (MOD) of immunostaining was measured using a Zeiss Axioskop microscope (Zeiss, Beijing, China), a 3-chip CCD camera C5810, (Hamamatsu, Beijing, China) and a camera control unit (Hamamatsu). Immunopositivity and immunonegativity were determined using a linear discriminant analysis method.

Fluorescence in situ hybridization (FISH) protocol. The 5- $\mu \mathrm{m}$ $\mathrm{PCa}$ tissue slides were deparaffinized, treated with $0.2 \mathrm{~N} \mathrm{HCl}$, incubated in $1 \mathrm{M}$ sodium thiocyanate and immersed in protease solution (Vysis Inc., Downers Grove, IL, USA) for $10 \mathrm{~min}$ at $37^{\circ} \mathrm{C}$. The tissues were then fixed with $10 \%$ formalin for $10 \mathrm{~min}$, denatured for $5 \mathrm{~min}$ at $72^{\circ} \mathrm{C}$ and sequentially incubated in 75, 90 and 100\% ethanol. The tissues were then treated with proteinase $\mathrm{K}$ for $6 \mathrm{~min}$ at $37^{\circ} \mathrm{C}$, followed by their dehydration and hybridization.

Next, two-colored FISH was performed, as described previously (13), using spectrum orange-labeled AR and spectrum green-labeled X-chromosome centromere region DNA probes (Vysis). Briefly, tissue array sections were hybridized with $3 \mu \mathrm{l}$ of each probe and Cot1-DNA ( $1 \mu \mathrm{g} / \mu \mathrm{l}$; Vysis) overnight at $37^{\circ} \mathrm{C}$ in a humidified atmosphere. The slides were then washed for counterstaining with $0.2 \mu \mathrm{M} 4$ ',6'-diamidino-2-phenylindole hydrochloride. FISH signals were scored with a fluorescence microscope (Zeiss) equipped with two double-band pass filters using 40X and 100X objective lenses. The number of AR gene signals and $\mathrm{X}$ centromere signals was evaluated by visual analysis of 800 to 1,200 nuclei per specimen. AR amplification was present if the AR to X ratio exceeded 1.5 (14), and $\mathrm{X}$ polysomy was present if the number of $\mathrm{X}$ centromere signals exceeded an average of 2 signals per cell (15).

Statistical data analysis. Medians and interquartile ranges are used to describe the data. Optical density is presented 
Table II. Clinical characteristics and survival of specimens with amplified and non-amplified AR.

\begin{tabular}{lcc}
\hline Entry & Non-amplified AR & Amplified AR \\
\hline Number of specimens & 24.0 & 13.00 \\
Mean age of Spc \pm SD & $73.40 \pm 8.40$ & $73.10 \pm 7.10$ \\
Mean GS \pm SD & $8.90 \pm 0.70$ & $9.00 \pm 0.60$ \\
Mean PSA \pm SD & $53.20 \pm 88.80$ & $30.10 \pm 46.60$ \\
Mean MOS B/w AD + TA \pm SD & $36.00 \pm 21.80$ & $33.10 \pm 11.60$ \\
Mean survival A/f AD (MOS) \pm SD & $52.80 \pm 28.50$ & $51.50 \pm 13.90$ \\
Mean AR expression (MOD) \pm SD & $0.24 \pm 0.10$ & $0.36 \pm 0.07$
\end{tabular}

AR, androgen receptor; Spc, specimen; SD, standard deviation; PSA, prostate-specific antigen (ng/ml); GS, gleason sum; MOS, medical outcome study; $\mathrm{B} / \mathrm{w}$, between; $\mathrm{AD}$, androgen deprivation; $\mathrm{TA}$, tissue acquisition; a/f, after; MOD, mean optical density.

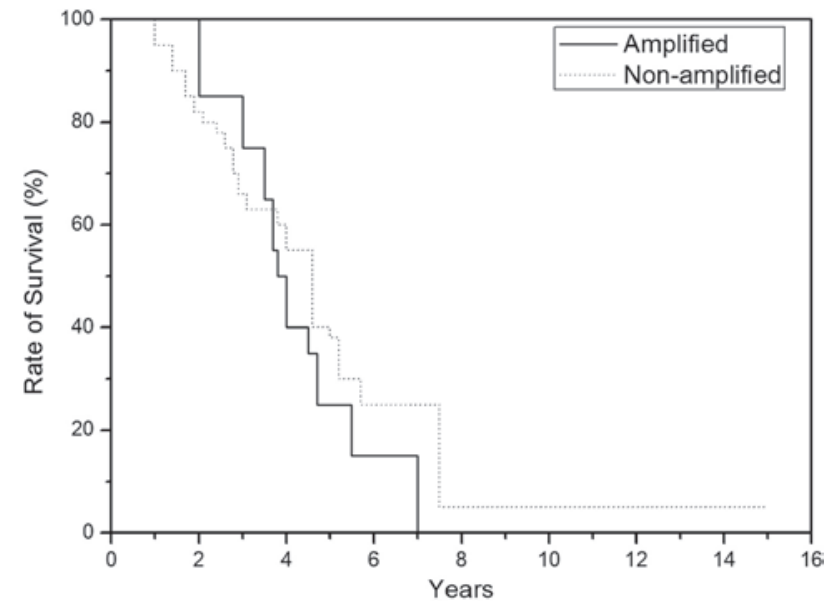

Figure 1. Comparative study of survival rates of amplified and non-amplified androgen receptor patients using a Kaplan-Meier plot.

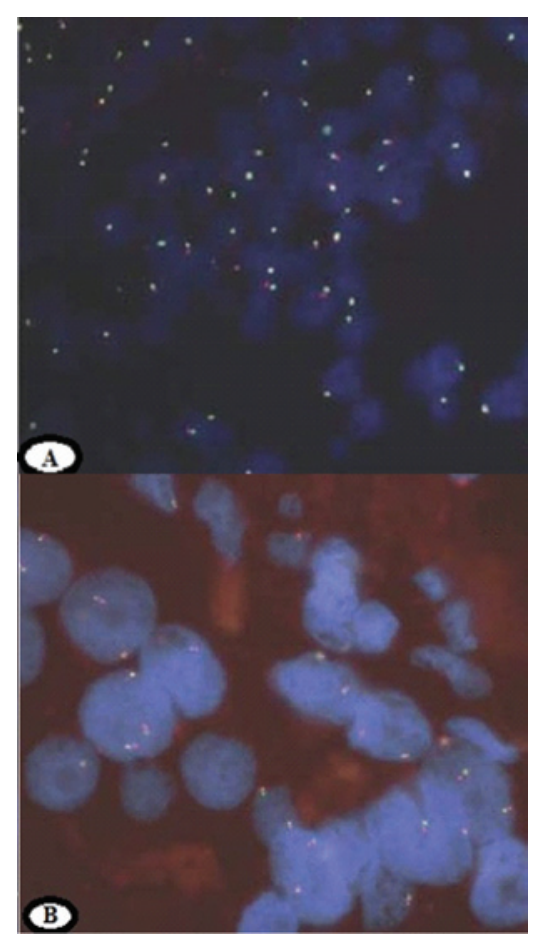

Figure 2. Representative fluorescence in situ hybridization (FISH) photomicrographs of (A) normal androgen receptor (AR) and (B) amplified AR. Sections of the specimens were stained with DAPI and analyzed by FISH. Red-labeled AR gene and green-labeled X-chromosome signals were observed.

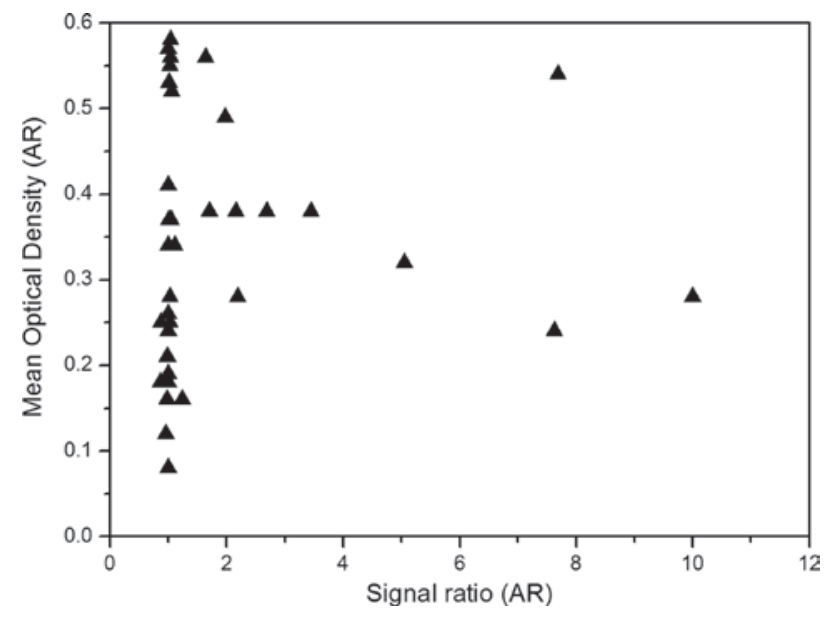

Figure 3. Comparative study of signal ratio (AR) and mean optical density of AR expression. AR, androgen receptor.

as the mean \pm standard deviation. The data were not evenly distributed, so a Wilcoxin analysis was used for comparison of the groups. Kaplan-Meier plot was used for comparison of the survival rates via log-rank analysis.

\section{Results}

Immunohistochemistry image analysis. The clinical characteristics and mean optical density of AR expression for the 37 patients are summarized in Table I. Out of 37 subjects with PCa recurrence, 13 (35\%) exhibited amplified AR expression, while 24 did not (Table I). Amplified AR (optical density, $0.45 \pm 0.03$ ) was more intensely immunostained than non-amplified AR (optical density, $0.21 \pm 0.06$ ) in the chosen tumor specimens. The intensity of AR immunostaining and the degree of AR amplification did not exhibit any association or trend. There was additionally no association between the protein expression of AR and the X-chromosome copy number.

No statistically significant differences were observed between amplified AR and non-amplified AR tumors specimens with regard to serum PSA levels, clinical tumor-node-metastasis stage, Gleason sum, time from androgen deprivation therapy to recurrence and survival following androgen deprivation (Table II and Fig. 1).

Additionally, no association with $\mathrm{X}$ polysomy was observed for these clinical parameters. Nevertheless, 6 of the 
recurrent PCa specimens (16\% of the chosen patients) exhibited X-chromosome 2.5 (copy number) and greater, while no differences were found when clinical characteristics between these groups were compared.

FISH analysis. AR FISH analysis results for tumors with normal AR and AR amplification are shown in Fig. 2A and B, respectively. The majority of the nuclei in the normal cells showed one green and one red signal, indicating that each nucleus had one $\mathrm{X}$ chromosome with one AR gene (Fig. 2A). The presence of two red signals in the FISH section in the majority of the PCa cells indicated the presence of AR gene amplification (Fig. 3).

\section{Discussion}

Since an effective therapy for the treatment of PCa recurrence has yet to be identified, an improved understanding of the mechanism behind the transition from androgen-dependent PCa to PCa recurrence may provide novel treatment targets (16). The only possible explanation for $\mathrm{PCa}$ recurrence is that the increased expression of AR protein through AR amplification may allow the expression of androgen-regulated genes despite castration levels of serum androgens. The present study found that the AR gene was amplified in $\sim 35 \%$ of $37 \mathrm{PCa}$ recurrence specimens; these results are far indicate an improved outcome compared with previous studies showing AR amplification in $23 \%$ of 47 (13), 25\% of 16 (17), 28\% of 54 (18), 30\% of 23 (5) and $31 \%$ of 13 (19) patients. The results compared AR expression levels between tumors with AR amplification and with a single AR signal. Reverse transcriptase polymerase chain reaction was previously used in 13 patients to demonstrate that AR mRNA expression occurred in the recurrence of 4 tumors and demonstrated that AR amplification was higher than AR mRNA in the recurrence of 9 tumors that were not amplified (19). When immunohistochemistry was used to compare AR protein expression between amplified and non-amplified tumors, the study by Visakorpi et al (5) found that $\sim 80 \%$ of primary tumor cells (as well as recurrence) expressed nuclear AR protein, but that there was no significant difference in the level of protein expression in primary tumors compared with other tumors (exhibiting recurrence or recurrence with AR amplification). In another study, the hybridization intensity for AR mRNA (ISH for 5 PCa reccurence specimens) was higher than that of the original PCa non-amplified specimens (18). The results showed different results for the length of survival in patients with advanced PCa treated with androgen deprivation based on the amplified or non-amplified AR in recurrent tumors. However, the present study found that amplified AR had no association with the duration of survival following androgen deprivation (Fig. 1), and no association was found between survival times following androgen deprivation and $\mathrm{X}$ polysomy. Koivisto et al reported that AR amplification occurred more often in males who exhibited a complete response to or longer interval between androgen deprivation and recurrence (18). However, the present study found no difference in the interval between androgen deprivation and recurrence. Recently, FISH was used to appraise the attainability of characterizing gene copy number alterations of circulating tumor cells isolated using the Cell Search system in specimens with PCa (particularly progressive castration-resistant metastatic PCa) (20); this study reported high-level chromosomal amplification of AR in $38 \%$ of the analyzed samples and relative gain of MYC in 56\% of the samples, which also supports the present results.

Immunohistochemical detection was also used to optimize the production of the linear association between AR protein and its DAPI immunostaining (21) using automated digital video image analysis for precise results. It was observed that those patients whose tumors demonstrated AR amplification exhibited a 5-month faster recurrence than those whose had non-amplified tumors. The amplified AR PCa recurrent tumors exhibited greater levels of AR protein expression, but this was not associated with survival. It was also demonstrated that AR protein expression was $60 \%$ higher in tumors with an AR copy number $>2.1$. Additionally, the study found that $\mathrm{X}$-chromosome copy number was increased in up to $13.8 \%$ of cancer specimens, which corresponds with the fact that AR copy number can be increased by X-chromosome polysomy, but will not impact on AR protein expression $(5,15,21)$.

In conclusion, this study is the first of its type, quantitatively comparing AR protein expression and AR amplification in PCa recurrence. This study demonstrated that AR influences tumor growth and progression even where androgen is deprived. Furthermore, the results indicated a potential contribution of AR amplification to AR activation in the relative absence of androgen.

\section{Acknowledgements}

The authors would like to thank the staff of the Wenzhou Central Hospital for their assistance in subject recruitment throughout the study period.

\section{References}

1. Lassi K and Dawson NA: Update on castrate-resistant prostate cancer: 2010. Curr Opin Oncol 22: 263-267, 2010.

2. Jemal A, Siegel R, Xu J and Ward E: Cancer statistics, 2010. CA Cancer J Clin 60: 277-300, 2010

3. Shahrokh F, Shariat, Axel S, et al: Tumor markers in prostate cancer I: Blood-based markers. Acta Oncol 50: 61-75, 2011.

4. Culig Z, Hobisch A, Bartsch G and Klocker H: Androgen receptor - an update of mechanisms of action in prostate cancer. Urol Res 28: 211-219, 2000

5. Visakorpi T, Hyytinen E, Koivisto $\mathrm{P}$, et al: In vivo amplification of the androgen receptor gene and progression of human prostate cancer. Nat Genet 9: 401-406, 1995.

6. Cude KJ, Montgomery JS, Price DK, et al: The role of an androgen receptor polymorphism in the clinical outcome of patients with metastatic prostate cancer. Urol Int 68: 16-23, 2002.

7. Heinlein CA and Chang C: Androgen receptor in prostate cancer. Endocr Rev 25: 276-308, 2004.

8. Chen CD, Welsbie DS, Tran C, et al: Molecular determinants of resistance to antiandrogen therapy. Nat Med 10: 33-39, 2004.

9. Gelmann EP: Molecular biology of the androgen receptor. J Clin Oncol 20: 3001-3015, 2002.

10. Shaffer DR and Scher HI: Prostate cancer: a dynamic illness with shifting targets. Lancet Oncol 4: 407-414, 2003.

11. Linja MJ and Visakorpi T: Alterations of androgen receptor in prostate cancer. J Steroid Biochem Mol Biol 92: 255-264, 2004.

12. Kiessling A, Hogrefe C, Erb S, et al: Expression, regulation and function of the ISGylation system in prostate cancer. Oncogene 28: 2606-2620, 2009

13. Bubendorf L, Kononen J, Koivisto P, et al: Survey of gene amplifications during prostate cancer progression by high-throughout fluorescence in situ hybridization on tissue microarrays. Cancer Res 59: 803-806, 1999. 
14. Edwards J, Krishna NS, Mukherjee R, Watters AD Underwood MA and Bartlett JM: Amplification of the androgen receptor may not explain the development of androgen-independent prostate cancer. BJU Int 88: 633-637, 2001.

15. Koivisto P, Hyytinen E, Palmberg C, et al: Analysis of genetic changes underlying local recurrence of prostate carcinoma during androgen deprivation therapy. Am J Pathol 147: 1608-1614, 1995

16. Feldman BJ and Feldman D: The development of androgen-independent prostate cancer. Nat Rev Cancer 1: 34-45, 2001.

17. Hyytinen ER, Haapala K, Thompson J, et al: Pattern of somatic androgen receptor gene mutations in patients with hormone-refractory prostate cancer. Lab Invest 82: 1591-1598, 2002.
18. Koivisto P, Kononen J, Palmberg C, et al: Androgen receptor gene amplification: A possible molecular mechanism for androgen deprivation therapy failure in prostate cancer. Cancer Res 57: 314-319, 1997.

19. Linja MJ, Savinainen KJ, Saramäki OR, Tammela TL, Vessella RL and Visakorpi T: Amplification and overexpression of androgen receptor gene in hormone-refractory prostate cancer. Cancer Res 61: 3550-3555, 2001.

20. Leversha MA, Han J, Asgari Z, et al: Fluorescence in situ hybridization analysis of circulating tumor cells in metastatic prostate cancer. Clin Cancer Res 15: 2091-2097, 2009.

21. Brown RS, Edwards J, Dogan A, et al: Amplification of the androgen receptor gene in bone metastases from hormone-refractory prostate cancer. J Pathol 198: 237-244, 2002. 